south Asia may have been made possible by a willingness to experiment with new social forms and technological strategies, including radical changes in agriculture and cuisine. In today's globally connected world, failures tend to have ramifications well beyond local contexts, making the lessons of the past perhaps even more relevant.
Kathleen D. Morrison is in the Department of Anthropology, University of Chicago, 1126 East 59th Street, Chicago, Illinois 60637, USA. e-mail: morrison@uchicago.edu

1. Green, R. C\& Weislec, M. L Asian Pespect.41,213-241(2002)

2. Hunt, T.L. \& Lipo, C P.Science 311, 1608-1606 (2006).

3. Flenley, I. R. \& King, S.M. Nature 307, 47-50 (1984).

4. Dransfield, Let al. Nature312, 750-752(1984)

\title{
FLUID DYNAMICS
}

\section{The rough with the smooth}

\section{Kwing-So Choi}

\section{Those who go with the flow assert that rough surfaces cause turbulence in fluids passing over them. The claim that, under certain conditions, the opposite is possible disturbs that cherished belief.}

Have you noticed how slick the exteriors of cars have become lately? Side mirrors are aerodynamically shaped; windows are an integral part of the doors; no parts hang out over the side of the body. Even windscreen wipers can be retracted when not in use. A smooth body is not only better looking; it can reduce aerodynamic drag, and thus makes a car faster and more fuel-efficient - or so we are led to believe. In fact, this prevailing wisdom may not be true: Fransson et al. ${ }^{1}$ report in Physical Review Letters that surface bumps are actually useful for reducing drag. So what's going on?

To get to the bottom of this matter, we must look at the evolution of fluid flows over a solid surface. Initially, these flows are silky smooth and move together; they are 'laminar'. Soon, however, the inherent instability of a laminar flow leads to its becoming random and chaotic, or 'turbulent.' Turbulent flow is usually associated with characteristics such as strong mixing of the fluid and high energy loss through dissipation. The drag that cars experience is therefore greater when the surrounding flow is turbulent: if a laminar flow can be maintained, the frictional drag acting on the car is much less.

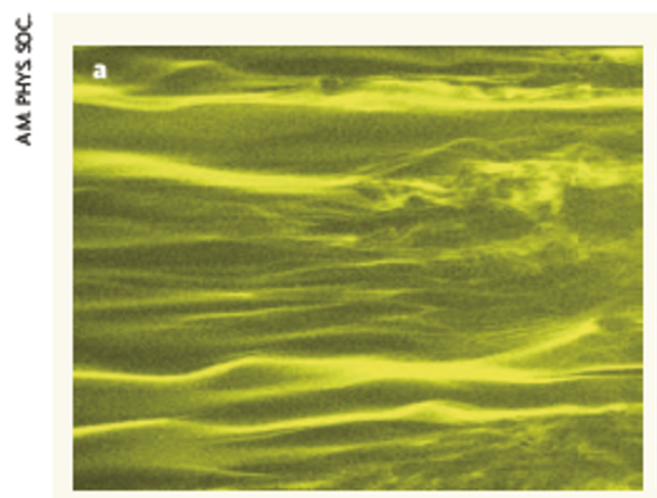

Figure 1| Flow patterns vis ualized by smoke. a, Laminar flow goes through the transition to turbulence without surface roughness. b, Fransson et al. ${ }^{1}$ maintain laminar flow for longer with a rough surface that creates regular disturbances.
Using surface roughness to reduce friction drag is, in the case of turbulent flow, not new. Randomized, V-shaped roughness elements are known to reduce friction drag ${ }^{4}$ in such flows, as are sand grains attached to a surface ${ }^{5}$. Cross-flow variation - a disturbance in velocity that occurs at right angles to the main flow - is also known to be caused by regular roughness elements. Its effect on turbulent flow has been studied theoretically ${ }^{6}$ by solving the equations governing fluid flows directly by computer.

In these cases, the key to drag reduction is efficient modification of organized structures of various shapes that have been identified as supplying most of the energy in turbulent flows. It has been argued that randomized roughness elements destroy such structures ${ }^{4}$, whereas cross-flow variation stabilizes a flow ${ }^{6}$, thus preventing the structures' reproduction. A regular pattern of roughness elements that introduces such a cross-flow variation to a laminar flow is central to Fransson and colleagues' study'. Their success in reducing drag reinforces the idea that organized structures in turbulent flows are the remnants of laminar flow structures after they have undergone the transition to turbulence.

Several investigations have sought to repeat physical and numerical experiments by artificially introducing disturbances into the flow, but only a few ${ }^{7}$ have succeeded. This implies that such results might be sensitive to small variations in geometric and flow parameters. So how robust is the flow-control strategy proposed by Fransson and colleagues?

It is fair to say that, as the shape of the roughness elements is critically important in stabilizing laminar flow, totally different flow patterns might emerge even when their shape is changed only slightly. Applying the technique to high-speed flows might also present a problem, as the streaks will become unsteady at higher speed. Roughness elements will introduce additional drag as they disturb the flow, so any reduction in friction drag by roughness elements must compensate for this if a strategy is to have practical value.

The prize for overcoming such problems, and recognizing that smooth is not everything, is great. Its value lies in the ability to design and build fuel-efficient airplanes, quieter submarines and faster cars - both more easily and more cheaply.

Kwing-So Choi is in the School of Mechanical, Materials and Manufacturing Engineering, University of Nottingham, University Park, Nottingham NG7 2RD, UK.

\footnotetext{
1. Fransson, L H. M, Talamelli, A, Brandt, L. \& Cossu, C. Phys. Rex Lett. 96,064501 (2006).

2. Cossu, C. \& Brandt, L. Phys Fluids 14, L57-L60 (2002)

3 Andersson, P.etal. J. Fluid Mech 428, 29-60 (2001).

4. Sirovich, L\& Karisson, S. Nature 388,753-755 (1997)

5. Tani, I. Pooc Japan Acod B 64, 21-24 (1988).

6. Schoppa, W.\& Hussain F. Phys Fluids 10, 1049-1051 (1998)

7. lusa Get al. J. Fluid Mech 473, 23-58 (2002).
} 